\title{
Acciones para la prevención y control de la anemia por deficiencia de hierro en niños hasta cinco años
}

\section{Actions for prevention and control of iron-deficiency anemia in up to five-years old children}

\author{
Lisett Nancy Selva Suárez,' Alcides Abad Ochoa Alonso"' \\ ' Doctor en Medicina. Especialista de II Grado en Nutrición e Higiene de los Alimentos. \\ Programa Mundial de Alimentos. La Habana, Cuba. \\ "Doctor en Medicina. Especialista de II Grado en Administración de Salud. Doctor en \\ Ciencias de la Salud. Escuela Nacional de Salud Pública. La Habana, Cuba.
}

\section{RESUMEN}

En Cuba la deficiencia de hierro es la carencia específica de micronutrientes más extendida y la principal causa de anemia en lactantes, niñas y niños hasta cinco años, en especial los menores de 24 meses. Las provincias orientales son las de mayor prevalencia con predominio casi absoluto de casos ligeros. El propósito de este trabajo es exponer la implementación de forma secuencial de tres proyectos que se ejecutan entre organismos y organizaciones cubanos y agencias de Naciones Unidas, para contribuir a la reducción de la anemia en niños y niñas hasta cinco años. La implementación de los proyectos se desarrolló con un enfoque de mercadotecnia social. Se aplicaron métodos cuantitativos, cualitativos y la triangulación de sus resultados. Todos los proyectos incluyeron la capacitación a actores clave en temas de alimentación saludable, nutrición y prevención de la anemia y un amplio trabajo educativo con las familias. En dos de ellos se entregó un alimento fortificado con hierro y en el tercero, se distribuyó otro producto tangible: los micronutrientes en polvo "Chispitas". Este último, sin precedentes en el país. Se describen las acciones realizadas en la provincia Holguín en la implementación de los tres proyectos y se exponen los criterios de evaluación. Concluida la intervención, se espera haber fortalecido las capacidades para la prevención y control de la anemia en los 14 municipios de la provincia y haber reducido la frecuencia de anemia en el grupo diana del municipio Calixto García. 
Palabras clave: Deficiencia de hierro, anemia, prevención y control, mercadotecnia social, educación nutricional, capacitación, micronutrientes en polvos, "Chispitas".

\begin{abstract}
I ron deficiency is the most extended specific lack of micronutrients and the main cause of anemia in infants, boys and girls up to five years of age, particularly those aged under 24 months. The Eastern provinces exhibit the highest prevalence with almost absolute predominance of mild cases. The objective of this study is to show the sequential implementation of three projects that are jointly carried out by Cuban bodies and organizations and UN agencies, with a view to reducing anemia in boys and girls aged up to five years. The implementation of these projects had a social marketing approach. Quantitative and qualitative methods and the triangulation of their results were used. All the projects included training of key actors in healthy feeding, nutrition and prevention of anemia as well as extensive educational work with the families. Two projects delivered an iron-fortified foodstuff and the other one distributed an unprecedented tangible product known as powdered micronutrients "Sprinkles". The actions undertaken in Holguin province to implement the three projects and the evaluating criteria were described. After the intervention, it is expected that the capacities for prevention and control of anemia in the 14 municipalities of this province be strengthened and the frequency of anemia in the target group in "Calixto García" municipality be reduced.
\end{abstract}

Key words: Iron deficiency, anemia, prevention and control, social marketing, nutritional education, training, powdered nutrients, Sprinkles.

\title{
I NTRODUCCI ÓN
}

La anemia por deficiencia de hierro constituye un problema de Salud Pública mundial. ${ }^{1-6}$ Recientemente la Organización Mundial de la Salud posicionó a la deficiencia de hierro en el lugar siete dentro de los 10 factores de riesgo prevenibles de enfermedad, discapacidad y muerte.

En Cuba la deficiencia de hierro es la carencia específica de micronutrientes más extendida y constituye la principal causa de anemia en mujeres en edad fértil, embarazadas, lactantes y niñas y niños hasta cinco años, en especial los menores de 24 meses. ${ }^{1,7-9}$

Aunque en el país la anemia es fundamentalmente ligera, combatirla ha sido una prioridad para el gobierno cubano. Dentro del marco de las políticas sanitarias nacionales en 1997 se implementó un "Plan Nacional para la Prevención y Control de la Deficiencia de Hierro y la Anemia en la población cubana" con carácter multisectorial y coordinado por el sector de la salud, que desarrolló múltiples acciones para enfrentar este problema. En consecuencia, en los últimos años se ha observado una reducción de la frecuencia y severidad de la anemia en grupos de riesgo pero aún los resultados alcanzados están por encima de las metas de salud proyectadas para el año $2015 .{ }^{10}$ En el año 2008 el referido Plan se fortalece con nuevos aspectos y se

http://scielo.sld.cu 
establece el "Plan Integral para la Prevención y Control de la Anemia por Deficiencia de Hierro en Cuba"11 a desarrollarse en el período 2008-2015.

La lucha contra la anemia en todos estos años ha contado con el apoyo de agencias del Sistema de Naciones Unidas como el Programa Mundial de Alimentos (PMA), el Fondo de la Naciones Unidas para la Infancia y la Organización Panamericana de la Salud. En el caso de PMA este apoyo se concreta en la actualidad a través de tres proyectos o programas que focalizan sus acciones en niños y niñas de 0 a 5 años, con prioridad en los menores de 24 meses de las provincias orientales, identificadas como las más vulnerables del país. Entre los organismos e instituciones del gobierno y organizaciones no gubernamentales vinculados a estos proyectos están los ministerios de Salud Pública, Agricultura, Industria Alimentaria, Comercio Interior, Educación y, la Federación de Mujeres Cubanas (FMC), todos bajo la coordinación general del Ministerio de Comercio Exterior y la inversión extrnajera.

Los proyectos son:

1. Apoyo al Plan Nacional para la Prevención y Control de la Anemia en niños menores de 5 años de las cinco provincias orientales (2008-2012). Proyecto 10589. ${ }^{12}$

2. Apoyo a la lucha contra la anemia en grupos vulnerables en Cuba. Ventana temática: infancia, seguridad alimentaria y nutrición. ${ }^{1}$ I ncluye 20 municipios de las provincias orientales y 4 de Pinar del Río y además de niños extiende sus acciones hasta las embarazadas.

Ambos proyectos promueven el desarrollo de capacidades y despliegan acciones vinculadas a la promoción de la alimentación saludable y la prevención de la anemia, y al fortalecimiento del Sistema de Vigilancia Alimentaria y Nutricional. Además, tienen en común la entrega de un alimento fortificado con hierro y otros micronutrientes así como propiciar la disponibilidad y el acceso a diferentes alimentos que aportan hierro.

3. Uso de los micronutrientes en polvo (MNP) para la prevención y control de la anemia en niños de 12 a 24 meses residentes en el municipio Calixto García de la provincia Holguín, 2009-2011.

Esta intervención incluye la distribución y el consumo del producto tangible MNP conocidos como "Chispitas", y el fortalecimiento de la educación nutricional a la familia a través de actores clave de la comunidad. Es la primera vez que esta intervención se implementa en el país y se hace con el apoyo técnico y financiero del Programa Mundial de Alimentos.

Se utilizaron métodos cuantitativos y cualitativos, entre ellos, revisión bibliográfica y documental, encuestas, entrevistas en profundidad a informantes clave, observación, grupos focales, trabajo con expertos, discusiones grupales, lluvia de ideas y métodos de la estadística descriptiva. Se realizó la triangulación de sus resultados. En todos los momentos la investigación previó la observación de los principios éticos para las investigaciones médicas en seres humanos contemplados en la Declaración de Helsinki de la Asociación Médica Mundial.

La intervención se realizó con un enfoque de mercadotecnia social y conllevó un amplio trabajo de campo.

Los principales métodos utilizados fueron: 
La estrategia para la implementación de los tres proyectos en la provincia Holguín se sustentó en las siguientes premisas:

- Participación de los actores locales desde la etapa de diseño.

- Trabajo en equipo y pensamiento colectivo.

- Socialización del conocimiento.

- Empoderamiento del grupo diana primario y de los agentes de cambio.

- Compromiso del equipo.

- Compromiso político.

- Participación multidisciplinaria e intersectorial.

- Integración.

- Marcos legales para el sector de la salud y el Programa Mundial de Alimentos.

- Indicaciones metodológicas y programas nacionales de los sectores de la salud y de educación, de la FMC y del Sistema de Naciones Unidas.

- Criterios de eficiencia.

\section{MPLEMENTACI ÓN}

Las principales acciones ejecutadas hasta el 2010 en la provincia se describen a continuación:

- Constitución de equipos multidisciplinarios provincial y municipal y elaboración de su plan de acción.

- Validación de los instrumentos a aplicar en los equipos multidisciplinarios .

- Aplicación de instrumentos para el diagnóstico del conocimiento de actores clave en alimentación saludable y prevención de la anemia.

- Determinación de la percepción de riesgo y acciones preventivas en embarazadas y madres de niños hasta cinco años.

- Procesamiento y análisis de la información.

- Construcción de la línea de base del conocimiento según segmentos.

- Realización de cuatro talleres provinciales para la sensibilización, contextualización y planificación de acciones.

- Elaboración de la versión definitiva del proceso de capacitación y de comunicación social.

- Realización de cuatro talleres municipales de planificación y 14 de capacitación. 
- Desarrollo de las acciones de abogacía que propicien el posicionamiento de la intervención y el empoderamiento de los actores clave.

- Validación de los materiales para la estrategia de comunicación.

- Formación de 897 facilitadores para desarrollar la capacitación en cascada.

- Ejecución de la capacitación en cascada.

- Capacitación de 608 agentes de cambio para la intervención con MNP.

- Desarrollo del componente educativo con las familias.

- Elaboración del plan de seguimiento y evaluación provincial.

\section{EVALUACIÓN}

\section{De proceso}

Para evaluar el componente de capacitación se realizará una medición a medio término del conocimiento que sobre alimentación saludable y prevención de la anemia alcanzaron los actores clave.

Se realizará una evaluación intermedia de la prevalencia de anemia.

\section{De resultados}

La estrategia será evaluada a partir de los resultados que se alcancen en las siguientes dimensiones y variables:

1. Dimensión Implementación.

- Intersectoriales: construcción de capacidades para el trabajo intersectorial, abogacía y concertación, alianzas estratégicas, el cambio de conducta respecto a las medidas para prevenir la anemia en la población meta y el posicionamiento y empoderamiento por parte de los actores.

- Sector de la salud: reforzamiento al interior del sector de los aspectos pertinentes señalados para el trabajo intersectorial, definición de prioridades de acciones y coordinaciones, estructura organizacional, capacitación y desarrollo de los recursos humanos, sistemas de información y vigilancia

\section{Dimensión Efectividad.}

- Reducción de la anemia observada a partir de la línea de base.

- Creación o fortalecimiento de las capacidades para la prevención y control de la anemia.

3. Dimensión Eficiencia.

- Costo unitario, costo beneficio.

http://scielo.sld.cu 
4. Dimensión Aceptabilidad social.

- Percepción y satisfacción de la población beneficiaria.

- Pertinencia de la intervención.

- Utilidad metodológica de la estrategia para el nivel local.

5. Dimensión Sostenibilidad.

- Política y social.

- Técnica.

- Financiera.

\section{De impacto}

Cambios en los valores de hemoglobina en relación con la línea de base.

La integración e implementación secuencial de estos tres proyectos propicia la incorporación de los siguientes factores críticos para el éxito: las lecciones aprendidas, las buenas prácticas, la armonización de iniciativas y la creación de sinergias internas y externas. Estos factores contribuirán a elevar la eficiencia del proceso al evitar la duplicación de esfuerzos en el diseño e implementación y en la utilización de recursos humanos y financieros.

Una vez concluida la intervención se espera haber reducido la frecuencia de anemia en el grupo diana y haber creado o fortalecido las capacidades para la prevención y control de la anemia en los 14 municipios de la provincia.

\section{REFERENCI AS BI BLI OGRÁFICAS}

1. Naciones Unidas [Internet]. Programa conjunto. Apoyo a la lucha contra la anemia en grupos vulnerables en Cuba. New York: NN.UU.; 2009 [citado 20 Oct 2010]. Disponible en: https://coin.fao.org/cms/media/2 L12565779790190/cuba_pc_apoyo_a_la_lucha_contra_la_anemia_2009.pdf

2. Baker Greer RD, Frank R. The Committee of Nutrition. Clinical reports diagnosis and prevention of iron deficiency and iron-deficiency anemia in infants and young children (0-3 years of age). Pediatrics [Internet]. 2010 [citado 20 Oct 2010].

Available in: http://pediatrics. aappublications.org/cgi/reprint/peds. 2010-2576v1

3. Freyre WB. La anemia por deficiencia de hierro: Estrategias de la OPS/OMS para combatirla. Salud Pública Mex. 1998;40: 199-205.

4. Stanco Gilda G. Funcionamiento intelectual y rendimiento escolar en niños con anemia y deficiencia de hierro [Internet]. COlombia: Sociedad Latinoamericana de Nutrición, Capítulo venezolano; 2010 [citado 25 oct 2010]. Disponible en:

http://colombiamedica. univalle. edu.co/Vol38N\% B01\%20supl/html/v38n1sla4.html

http://scielo.sld.cu 
5. OMS/UNICEF. Declaración conjunta. La anemia como centro de atención. Hacia un enfoque integrado para el control eficaz de la anemia. Ginebra: OMS; 2004.

6. UNICEF [Internet]. Tracking Progress on Child and Maternal Nutrition. A survival and development priority. Santiago de Chile: UNICEF; 2009 [citado 26 oct 2010]. Available in:

http://www.unicef.pt/docs/Progress_on_Child_and_Maternal_Nutrition_EN_110309.p $\underline{\mathrm{df}}$

7. Reboso Pérez J, Cabrera Núñez E, Pita Rodríguez G, Jiménez Acosta S. Anemia por deficiencia de hierro en niños de 6 a 24 meses y de 6 a 12 años de edad. Rev Cubana Salud Pública. 2005; 31(4): 306-12.

8. Gay Rodríguez J. Anemia nutricional en un grupo de niños aparentemente sanos de 2 a 4 años de edad. Rev Cubana Aliment Nutr. 2002;16(1):31-4.

9. Gay Rodríguez J, Padrón Herrera M, Amador M. Prevención y Control de la anemia y la deficiencia de hierro en Cuba. Rev Cubana Aliment Nutr. 1995;9(1).

10. Ministerio de Salud Pública de Cuba. Proyecciones de la Salud pública en Cuba para el 2015. La Habana: MINSAP; 2006.

11. Ministerio de Salud Pública de Cuba. Plan integral para la prevención y el control de la anemia por deficiencia de hierro en Cuba. La Habana: MINSAP; 2008.

12. Programa Mundial de Alimentos [Internet]. Proyecto de Desarrollo Cuba 10589 Apoyo al Plan Nacional para la prevención y el Control de la Anemia en las cinco provincias orientales de Cuba. Roma: Programa; Oct 2007 [citado 25 Oct 2010]. Disponible en: http://www.onu.org.cu/pma/proyectos.asp

Recibido: 28 de octubre de 2010.

Aprobado: 9 de noviembre de 2010.

Lisett Nancy Selva Suárez. Programa Mundial de Alimentos. Calle 36 No. 724 e/ 7ma. y 17. Playa. La Habana, Cuba.

lisette.selva@wfp.org, alcidesochoa@infomed.sld.cu 